\title{
Estudo das Associações Genéticas entre Perímetro Escrotal e Características Reprodutivas de Fêmeas Nelore ${ }^{1}$
}

\section{Selmos Luiz Gressler², José Aurélio Garcia Bergmann ${ }^{3}$, Carmen Silva Pereira ${ }^{3}$, Vânia Maldini Penna $^{3}$, Jonas Carlos Campos Pereira ${ }^{3}$, Maria Gizelma de Menezes Gressler ${ }^{4}$}

\begin{abstract}
RESUMO - Os objetivos deste trabalho foram estimar parâmetros genéticos para as características perímetro escrotal (PE), medido aos $12(\mathrm{PE} 12, \mathrm{n}=652)$ e $18(\mathrm{PE} 18, \mathrm{n}=607)$ meses de idade, idade ao primeiro parto (IPP, $\mathrm{n}=1582)$, datas do primeiro $(\mathrm{DPP}, \mathrm{n}=1582)$ e segundo partos (DSP, $n=644$ ) e primeiro intervalo de partos (PIP, $n=644$ ) de animais da raça Nelore. Os parâmetros foram estimados pelo método da máxima verossimilhança restrita, usando-se um modelo animal e incorporando 15.648 informações de pedigree. As estimativas de herdabilidade das análises incluindo somente uma variável foram $0,24 \pm 0,10 ; 0,31 \pm 0,10 ; 0,01 \pm 0,03 ; 0,11 \pm 0,05 ; 0,07 \pm 0,08$; e $0,10 \pm 0,10$, respectivamente, para PE12, PE18, IPP, DPP, DSP e PIP. Estas estimativas foram semelhantes às obtidas nas análises em que foram incluídas duas variáveis, com exceção da análise incluindo os dois PE (0,68 e 0,61 para PE12 e PE18, respectivamente). As estimativas de correlações genéticas entre PE12 e IPP, DPP, DSP e PIP foram, respectivamente, - 1,$00 ;-0,08 ;-0,71$; e $-0,37$. Por outro lado, as correlações genéticas entre PE18 e as características observadas nas fêmeas foram, na mesma ordem, $-1,00 ; 0,21 ;-0,35 ;$ e $-0,44$. A seleção para o PE12 seria mais efetiva que a seleção para o PE18, quando se deseja obter melhorias relacionadas às características reprodutivas das fêmeas.
\end{abstract}

Palavras-chave: correlação genética, reprodução

\section{Genetic Association among Scrotal Circumference and Female Reproductive Traits in Nellore}

\begin{abstract}
The objectives of this work were to estimate of genetic parameters for scrotal circumference (SC) at 12 (SC12, $\mathrm{n}=652)$ and $18(\mathrm{SC} 18, \mathrm{n}=607)$ months of age, age at first calving $(\mathrm{AFC}, \mathrm{n}=1,582)$, first $(\mathrm{FCD}, \mathrm{n}=1,582)$ and second $(\mathrm{SCD}, \mathrm{n}=644)$ calving dates and first calving interval (FCI, $\mathrm{n}=644$ ) in Nellore cattle. Parameters were estimated using REML methodology, with animal models and considering the relations among 15,648 animals. Heritability estimates from univariate analyses were $.24 \pm .10, .31 \pm .10$, $.01 \pm .03, .11 \pm .05, .07 \pm .08$ and $.10 \pm .10$, respectively, for SC12, SC18, AFC, FCD, SCD and FCI. These values were close to the estimates from bivariate analyses, except for the analysis including both SC (heritability estimates of .61 and .68, respectively, for SC12 and SC18). Genetic correlation estimates between SC12 and, respectively, AFC, FCD, SCD and FCI were -1.00, -.08, -.71, and -.37. In the same order, genetic correlation between SC18 and female reproductive traits were $-1.00, .21,-.35$ and -.44 . The selection for SC12 would be more effective than selection for SC18 when trying to improve the reproductive traits of the females.
\end{abstract}

Key Words: genetic correlation, reproduction

\section{Introdução}

Os trabalhos disponíveis na literatura brasileira relativos à avaliação da eficiência reprodutiva da fêmea zebu têm enfatizado quase exclusivamente a idade à primeira cria e o intervalo de partos (ANDRADE,1991). Embora possam ser utilizadas para expressar a qualidade reprodutiva dos rebanhos, essas duas características podem não ser as mais adequadas para a seleção e o descarte das fêmeas bovinas de corte.

Duas outras características, data do parto e/ou dias para o parto, têm sido recomendadas para avaliação do desempenho reprodutivo das fêmeas bovinas de corte (BERGMANN, 1993). Essas características são indicadoras da habilidade individual das vacas para conceberem cedo na estação de monta e parirem cedo na estação de nascimento. Segundo MORRIS e CULLEN (1988) e MORRIS (1990), o intervalo de partos não seria uma característica importante, quando

\footnotetext{
${ }^{1}$ Parte da Dissertação de Mestrado do primeiro autor apresentada à Escola de Veterinária da UFMG. Pesquisa parcialmente financiada pelo CNPq, FAPEMIG, PRPq-UFMGeFEP/MVZ.

2 Mestre em Zootecnia, Fazenda Minuano, Dourados - MS. E.mail: selmos@zaz.com.br

3 Professor, Bolsista do CNPq, Departamento de Zootecnia da Escola de Veterinária da UFMG - Caixa Postal 567 - CEP: 30.161-970 - Belo Horizonte, MG

${ }^{4}$ Médico-Veterinário, Fazenda Minuano, Dourados - MS
} 
estações de monta de curta duração são adotadas, em razão de sua correlação negativa com a data do parto.

Apesar da seleção para aumento do perímetro escrotal não trazer benefício econômico direto, este está geneticamente correlacionado com várias características reprodutivas de machos e fêmeas (BERGMANN, 1993) e com características ponderais (BERGMANN et al., 1996). Ademais, o perímetro escrotal apresenta alta herdabilidade (QUIRINO e BERGMANN, 1998, entre outros) e tem mensuração fácil e de baixo custo. Assim, sua inclusão tem sido recomendada nos programas de melhoramento genético para a eficiência reprodutiva em bovinos de corte (BOURDON e BRINKS, 1986; BERGMANN, 1993; e NOTTER, 1995). Estudos em países de clima temperado demonstraram que uma única medição do perímetro escrotal, próximo aos 12 meses de idade, seria suficiente para caracterizar o desenvolvimento testicular de tourinhos de raças européias (TOELLE e ROBISON, 1985; e NOTTER, 1988).

No Brasil, trabalhos abordando a associação entre perímetro escrotal e características reprodutivas das fêmeas zebus são poucos. Para orientação segura às freqüentes indagações de produtores relativas à idade mais eficiente para se medir o perímetro escrotal e aos possíveis efeitos da seleção para o perímetro escrotal sobre o desempenho reprodutivo de fêmeas, faz-se necessário estudos que abordem aspectos genéticos do perímetro escrotal e das características reprodutivas de fêmeas zebus.

Foram objetivos desse trabalho a obtenção de estimativas de herdabilidades para as características perímetro escrotal aos 12 e aos 18 meses de idade, data do primeiro e do segundo partos, idade ao primeiro parto e primeiro intervalo de partos, de animais da raça Nelore submetidos à estação de monta restrita, e caracterizar as associações genéticas e fenotípica entre estas características.

\section{Material e Métodos}

Foram utilizadas as informações de 652 machos, nascidos de 1991 a 1995 , e de 1582 fêmeas, nascidas no período de 1978 a 1993, filhos de, respectivamente, 87 e 192 touros, pertencentes ao rebanho Nelore da Colonial Agropecuária Ltda., Janaúba, MG. A matriz de parentesco utilizada totalizou 15.648 animais diferentes, nascidos entre 1964 e 1995 . O rebanho era constituído de animais puros de origem, acasalados por monta natural, ou IA, sob regime de estação de monta desde 1978, cuja duração variou de 100 a 141 dias entre os anos, tendo seu início no mês de dezembro e seu término nos meses de abril ou maio.

Os bezerros foram criados até a desmama em pastagem não-irrigada. A partir de 1993, após a desmama, os bezerros passaram a ser recriados em pasto irrigado, de junho a novembro; as demais categorias de animais foram criadas em pastagem nãoirrigada. Apenas novilhas que atingiram peso próximo aos $300 \mathrm{~kg}$ no início da estação de monta permaneceram no rebanho. Além disso, as fêmeas com diagnóstico de gestação negativo após o término da estação de monta foram descartadas. Instalações e demais condições de manejo do rebanho seguiram recomendações utilizadas na criação extensiva de gado de corte.

As medidas de perímetro escrotal aos 12 e 18 meses de idade foram feitas transversalmente na região de maior diâmetro do escroto com a utilização de fita métrica metálica, em idades variando, respectivamente, de 277 a 457 dias (360 dias de média) e 458 a 638 dias (539 dias de média).

As características observadas nas fêmeas foram divididas em dois grupos; o primeiro relacionado ao início da vida reprodutiva (idade ao primeiro parto e a data do primeiro parto, $\mathrm{N}=1582$ ) e o segundo associado à periodicidade reprodutiva (data do segundo parto e primeiro intervalo de partos, $\mathrm{N}=644$ ). Conforme estudo realizado por NOTTER et al. (1993), optou-se por estudar a data do primeiro e segundo partos como duas características independentes. Para o cálculo das datas do primeiro e segundo partos, adotou-se a definição proposta por PONZONI (1992).

A data do parto, apesar de ser uma variável contínua, freqüentemente não apresenta distribuição normal (NOTTER e JOHNSON, 1987). Na tentativa de solucionar esse problema, diversos autores têm sugerido e/ou realizado transformações dos dados (NOTTER e JOHNSON, 1988; NOTTER et al., 1993). Entretanto, em outros estudos, MEYER et al. (1990) e PONZONI e GIFFORD (1994), ao realizarem comparações entre estimativas obtidas com dados transformados e não-transformados, não encontraram diferenças significativas. Provavelmente, em virtude disso, MEYER et al. (1991) e JOHNSTON e BUNTER (1996) não fizeram qualquer transformação nos dados, quando analisaram as características data do parto e dias para o parto. No presente estudo, análises preliminares foram realizadas utilizando-se o desvio da observação em relação à mediana para a característica (BOURDON e BRINKS, 1983; NOTTER et al., 1993). Os resultados dessas análises 
não diferiram dos obtidos a partir de análises com dados não-transformados. Por isso, optou-se, neste trabalho, pela não-transformação dos dados.

Foram considerados apenas os animais que tiveram partos inseridos no período das estações de nascimento e apenas as fêmeas que possuíam parto documentado na referida estação.

As análises estatísticas foram realizadas no Departamento de Zootecnia da Escola de Veterinária da UFMG. Foi utilizado o pacote estatístico SAS (1990) para a formação de arquivos, consistência e análise descritiva dos dados, assim como para a verificação dos fatores ambientes sobre as diversas características. Maior detalhamento sobre esses resultados foram publicados por BERGMANN et al. (1998).

Nas estimativas de herdabilidade e de correlações genéticas, foi usado o método de máxima verossimilhança restrita, utilizando-se modelo animal, com o aplicativo MTDFREML (BOLDMAN et al., 1995). Foram feitas análises envolvendo apenas uma variável e análises envolvendo duas variáveis (perímetro escrotal em uma das idades e cada uma das quatro características observadas nas fêmeas). Para os modelos com apenas uma variável, foi utilizada a versão que possibilita a obtenção de estimativas dos erros-padrão.

Os modelos adotados foram resultantes de investigações preliminares sobre a importância $(\mathrm{P}<0,05)$ dos diversos efeitos ambientes para as características (BERGMANN et al., 1998). Assim, os efeitos fixos considerados foram: ano e mês de nascimento (para as seis características); sexo do primeiro bezerro e mês do parto anterior (para data do segundo parto e o primeiro intervalo de partos); peso ao nascimento do primeiro bezerro como covariável, efeito linear (para idade ao primeiro parto e a data do primeiro parto); e idade ao parto como covariável, efeitos linear (para as datas do primeiro e do segundo partos e para o primeiro intervalo de partos) e quadrático (para data do primeiro parto) e idade quando da mensuração, como covariável, efeitos linear (para o perímetro escrotal aos 12 e aos 18 meses de idade) e quadrático (para o perímetro escrotal aos 18 meses de idade).

\section{Resultados e Discussão}

\section{Análises envolvendo apenas uma variável}

$\mathrm{Na}$ Tabela 1, são apresentadas as estimativas dos componentes de variância e herdabilidade para as seis características estudadas. As estimativas de herdabilidade do perímetro escrotal aos 12 e aos 18 meses de idade foram inferiores às obtidas por BERGMANN et al. (1996) para a mesma fonte de dados. Cabe ressaltar que as amostras entre os dois estudos diferiram. Portanto, diferenças entre estimativas de herdabilidade podem ser decorrentes de diferenças entre amostras de dados analisados, as quais, por serem finitas, apresentam variações, provavelmente em decorrência de desvios de amostragem. Além disso, diferenças entre modelos de análise também levam a diferentes estimativas. Assim, MARTINS FILHO (1991) relatou ter encontrado, para o perímetro escrotal, desvios de amostragem entre dois trabalhos realizados a partir da mesma fonte de dados em animais da raça Nelore.

Entretanto, os resultados obtidos para o perímetro escrotal concordam com os trabalhos encontrados na literatura, com valores médios a elevados para estimativas de herdabilidade do perímetro escrotal, indicando a existência de variabilidade genética. A herdabilidade do perímetro escrotal aos 12 meses de idade, neste estudo, encontra-se próxima à estimativa encontrada por LÔBO et al. (1994) para animais da raça Nelore $(0,25)$. Para o perímetro escrotal aos 18 meses de idade, a herdabilidade encontrada foi intermediária entre as estimativas encontradas para a raça Nelore criada no Brasil (GRESSLER, 1998).

Contrastando as estimativas de herdabilidade para as duas idades, fica evidente a maior variabilidade genética para perímetro escrotal medido aos 18 meses, o que poderia indicar ser esta a idade mais adequada para seleção pelo perímetro escrotal dos machos Nelore, em regime de criação extensiva deste estudo. Entretanto, a diferença entre as duas estimativas de herdabilidades foi menor que a encontrada por BERGMANN et al. (1996).

A herdabilidade estimada para a idade ao primeiro parto (Tabela 1) está abaixo dos valores encontrados na literatura para animais Zebus (GRESSLER, 1998). Apesar de existente, a variação genética aditiva para a idade ao primeiro parto é menor que a variação genética residual. Segundo MILAGRES et al. (1979), as baixas estimativas de herdabilidades para características reprodutivas podem também ser conseqüência de redução na variação genética resultantes da seleção natural. Adicionalmente, a idade ao primeiro parto é uma característica muito dependente do manejo reprodutivo e da idade na qual as fêmeas são incorporadas no rebanho para reprodução (NOTTER, 1995). Segundo esse autor, esta característica está associada à idade a puberdade de fêmeas 
zebus apenas quando as novilhas são acasaladas próximo ou antes de dois anos de idade. A média de idade aproximada da entrada em reprodução no rebanho em estudo foi de 27 meses, quando praticamente todas as novilhas já tinham atingido a puberdade, e estavam aptas a conceber. Dessa forma, as variações na idade da concepção poderiam ser, provavelmente, decorrentes de amostragem, e não de diferenças genéticas.

As estimativas de herdabilidade para data do parto podem ter sido subestimadas no presente estudo, em virtude da exclusão das fêmeas que não obtiveram êxito em conceber e parir. MEACHAM e NOTTER (1987) e JOHNSTON e BUNTER (1996), entre outros, demonstraram preocupação com a eliminação das informações referentes as vacas vazias. Segundo esses autores, a eliminação de vacas vazias pode reduzir a expressão das diferenças devidas aos touros na habilidade reprodutiva das vacas remanescentes. A eliminação de fêmeas vazias, filhas de reprodutores específicos, pode resultar na subestimação dos parâmetros genéticos das características reprodutivas. No presente estudo, as infor- mações relativas a fêmeas não-gestantes ao final da estação de monta não estavam disponíveis e, por isso, não houve meios de se considerar este efeito.

A herdabilidade estimada para a data do primeiro parto $(0,11$; Tabela 1$)$ foi semelhante à descrita por KOOTS et al. (1994a). De forma similar, a herdabilidade estimada para a data do segundo parto $(0,07$; Tabela 1$)$ foi próxima às estimativas da literatura estrangeira. Não foram encontradas, na literatura nacional, estimativas de herdabilidade para essas duas características.

A menor estimativa de herdabilidade para a data do segundo parto em relação à data do primeiro parto está de acordo com os comentários de MEACHAM e NOTTER (1987), em que a menor herdabilidade da data do segundo parto pode ser reflexo do descarte de vacas vazias ou de mudanças reais na magnitude das variâncias genética e/ou ambiente. Ainda, segundo esses autores, a variação na data do primeiro parto poderia estar relacionada a diferenças entre as idades à puberdade dos animais, que deveriam ser menos importantes para a data do segundo parto. Por outro lado, variações no período de serviço influenci-

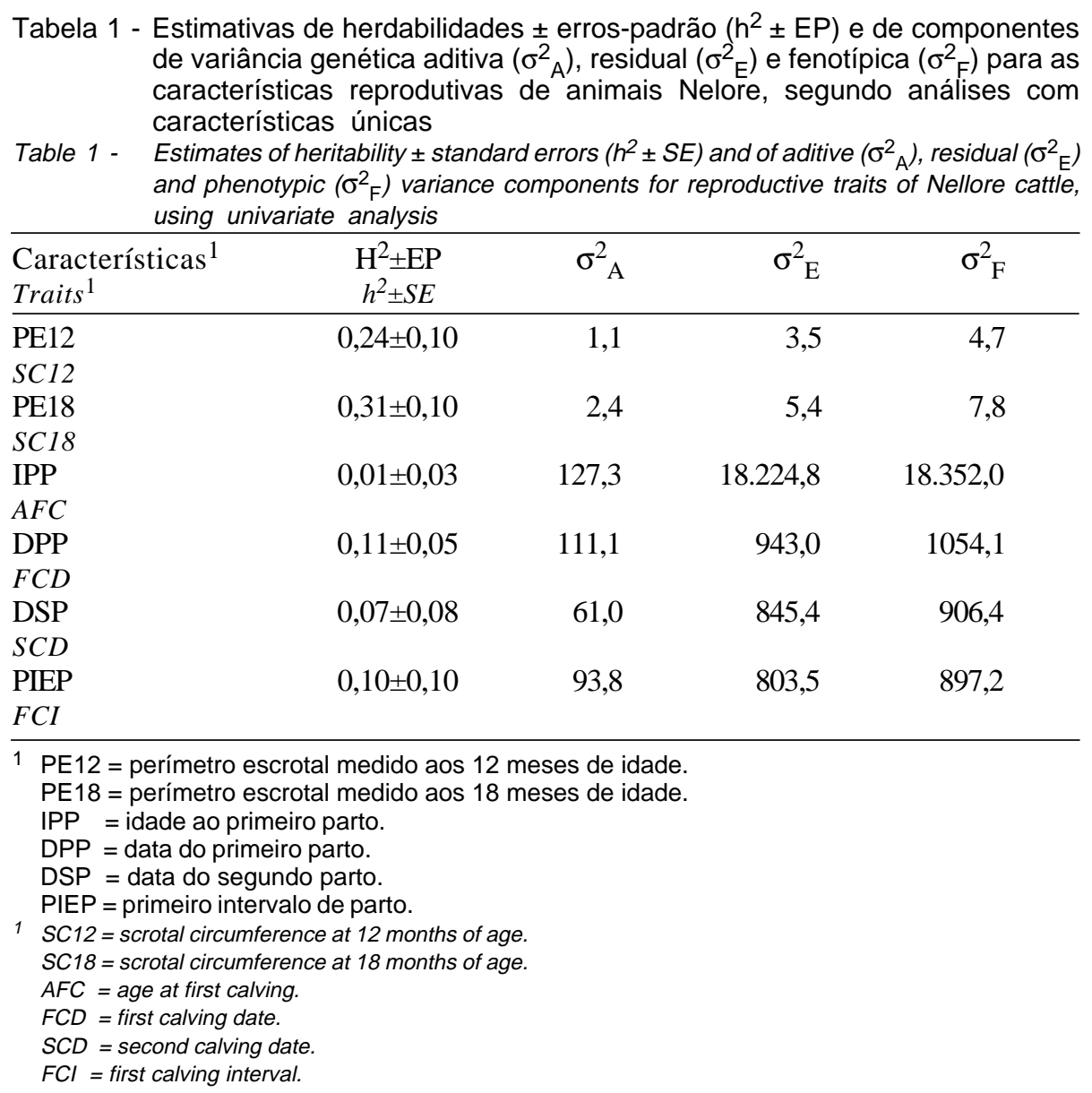


ariam apenas a data do segundo parto.

Para o primeiro intervalo de partos, a herdabilidade estimada na análise com uma variável $(0,10$; Tabela 1$)$ foi idêntica à média ponderada pelos erros-padrão citada por MERCADANTE (1995) para animais zebuínos, porém superior à média ponderada para animais taurinos (KOOTS et al. 1994a).

A estimativa de herdabilidade para o primeiro intervalo de partos foi semelhante à obtida para a data do primeiro parto e pouco superior à obtida para a data do segundo parto. Evidências encontradas na literatura (BERGMANN, 1993; LÔBO, 1996; e BERGMANN et al., 1998, entre outros) sugerem que o intervalo de partos apresenta maiores vícios e erros de mensuração e estimação que a data do parto. Em função disso, esperar-se-iam maiores diferenças entre as duas estimativas de herdabilidades. Poder-seia conjeturar que a inclusão de vacas que falharam em conceber refletiria em aumento dessas diferenças. Apesar disso, a seleção para a data do parto parece ser mais adequada, visto que apenas uma ocorrência de parto é necessária para se estimar o mérito genético dos animais, não sendo esse o caso para o intervalo de partos. De modo geral, as estimativas de herdabilidade encontradas nesse estudo estão de acordo com as conclusão de BOURDON e BRINKS (1983), de que o intervalo de partos ajustado para a data do parto anterior corresponde essencialmente à data do parto.

Os erros-padrão das estimativas de herdabilidade para as características reprodutivas das fêmeas foram altos e refletem falhas associadas à sua mensuração. Por outro lado, os erros-padrão associados às estimativas de herdabilidade dos perímetros escrotais foram menores (Tabela 1). Entretanto, para ambos os grupos de características, a magnitude desses erros está de acordo com a literatura (GRESSLER, 1998)

\section{Análises envolvendo mais de uma variável}

As estimativas dos componentes de (co)variância e dos parâmetros genéticos, obtidas a partir de análises com duas variáveis, são apresentadas nas Tabelas 2 e 3. Os componentes de variância estimados pelas análises com duas variáveis foram semelhantes aos das análises com uma variável (Tabela 1), exceção feita apenas para a análise envolvendo o perímetro escrotal aos 12 e 18 meses de idade, em que a variância genética aditiva foi maior e a variância residual, menor, resultando em estimativas de herdabilidades pelo menos duas vezes superiores. Variações nos parâmetros genéticos entre modelos com apenas uma variável e mais de uma variável foram também relatadas em outros estudos (MEYER et al., 1990; JOHNSTON e BUNTER, 1996). De acordo com FALCONER (1989), por reduzir a magnitude da variância residual, a inclusão de duas medidas aumentaria o valor proporcional da variância genética. Como a correlação genética entre as duas medidas do perímetro escrotal foi próxima da unidade (Tabelas 2 e 3), o aumento das estimativas de herdabilidade, nesse caso, poderia ser conseqüência do uso de medidas repetidas.

Para as demais características, as estimativas de herdabilidade obtidas pelos dois modelos variaram pouco. Para o perímetro escrotal medido aos 18 meses de idade, foram realizadas cinco análises com variáveis múltiplas. As herdabilidades estimadas variaram de 0,30 a 0,68 , com média de 0,38 . Para o perímetro escrotal, medido aos 12 meses de idade, as herdabilidades estimadas nas cinco análises variaram de 0,24 a 0,61, com média de 0,32. Em relação às características reprodutivas das fêmeas, as estimativas de herdabilidade foram semelhantes entre a análise única e as análises conjuntas com cada um dos perímetros escrotais.

As estimativas de correlações genéticas entre o perímetro escrotal nas duas idades e as características reprodutivas das fêmeas foram todas favoráveis, com exceção da correlação entre perímetro escrotal medido aos 18 meses de idade e a data do primeiro parto $(0,21)$. Também, as correlações fenotípicas foram todas em sentido favorável, mas de magnitude inferior às genéticas, exceção feita à correlação fenotípica entre o perímetro escrotal aos 18 meses e a data do primeiro parto. As correlações residuais não diferiram dos valores iniciais (inexistentes) para as características medidas em animais de sexos diferentes, o que está de acordo com os comentários de BOLDMAN et al. (1995). Para as análises envolvendo perímetros nas duas idades, a correlação residual foi positiva e elevada, indicando que o ambiente residual agiu de forma semelhante para as duas medidas do perímetro escrotal.

De modo geral, os resultados obtidos neste estudo para as correlações entre o perímetro escrotal e as características reprodutivas das fêmeas (Tabelas 2 e 3) foram semelhantes aos encontrados na literatura (GRESSLER, 1998). Para as correlações genéticas entre os perímetros escrotais e a idade ao primeiro parto, valores semelhantes foram citados em animais da raça Nelore por MARTINS FILHO (1991). As correlações genéticas entre o perímetro escrotal e a idade ao primeiro parto, observadas neste estudo, 
Tabela 2 - Estimativas dos componentes de (co)variância e dos parâmetros genéticos provenientes das análises com características múltiplas com o perímetro escrotal medido aos 12 meses de idade (característica I) e cada uma das seguintes características (2): idade ao primeiro parto (IPP), data do primeiro (DPP) e segundo partos (DSP), primeiro intervalo de partos (PIP) e perímetro escrotal aos 18 meses de idade (PE18) de animais Nelore

Table 2 - Estimates of variance and covariance components, and genetic parameters resulting from bivariate analysis including scrotal circumference at 12 months of age (trait 1) and each one of the following traits (2): age at first calving (AFC), first $(F C D)$ and second calving dates (SCD), first calving interval $(F C l)$ and scrotal circumference at 18 months of age (SC18) of Nellore cattle

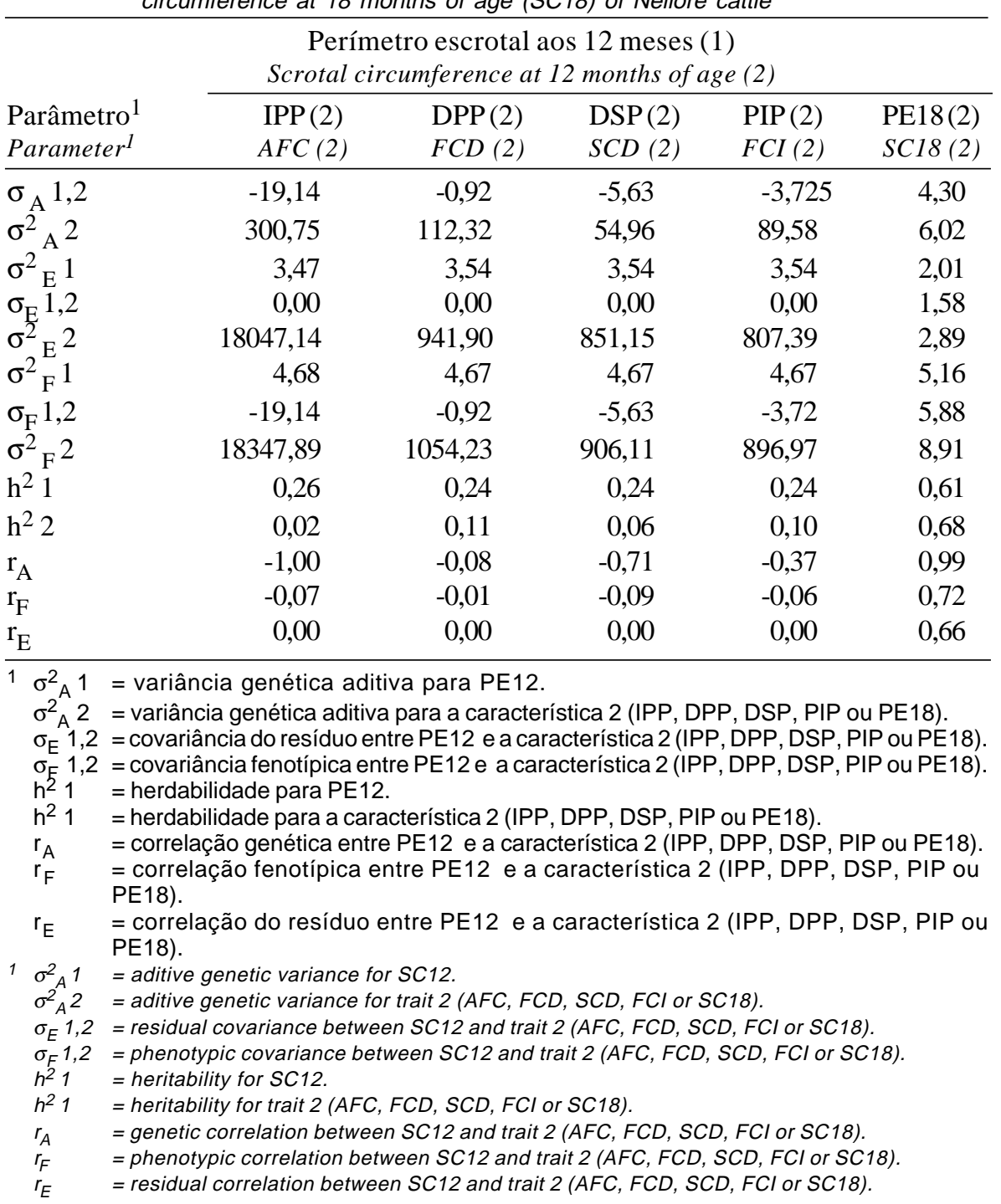

estão de acordo com as observações de BOLDMAN et al. (1995), segundo os quais, em conjuntos de dados com número de informações reduzido e envolvendo características observadas em sexos diferentes, existe tendência de convergência das correlações genéticas para valores próximos da unidade.

Para as correlações genéticas entre os perímetros escrotais e o primeiro intervalo de parto, os resultados deste estudo assemelham-se aos descritos para animais taurinos (GRESSLER, 1998), sendo estas mais altas para o perímetro escrotal medido aos 18 meses de idade. Como o primeiro intervalo de partos está relacionado com as datas do primeiro e segundo partos (BERGMANN et al., 1998), esta correlação poderia ser entendida como uma função das correlações entre as duas datas do parto e os perímetros escrotais. De fato, a correlação genética entre a data do primeiro parto e o perímetro escrotal medido aos 18 meses foi positiva, isto é, maior perímetro escrotal estaria associado ao atraso na data do primeiro parto e à tendência de redução no primeiro intervalo de partos. Por outro lado, a correlação negativa entre o perímetro escrotal aos 18 meses e a data do segundo parto significa que maiores períme- 
Tabela 3 - Estimativas dos componentes de (co)variância e dos parâmetros genéticos provenientes das análises com características múltiplas com o perímetro escrotal medido aos 18 meses de idade (característica I) e cada uma das seguintes características (2): idade ao primeiro parto (IPP), data do primeiro (DPP) e do segundo partos (DSP), primeiro intervalo de partos (PIP) e perímetro escrotal aos 12 meses de idade (PE12) de animais Nelore

Table 3 - Estimates of variance and covariance components, and genetic parameters resulting from bivariate analysis including scrotal circumference at 18 months of age (trait 1) and each one of the following traits (2): age at first calving (AFC), first (FCD) and second calving dates (SCD), first calving interval (FCI) and scrotal circumference at 12 months of age (SC12) of Nellore cattle

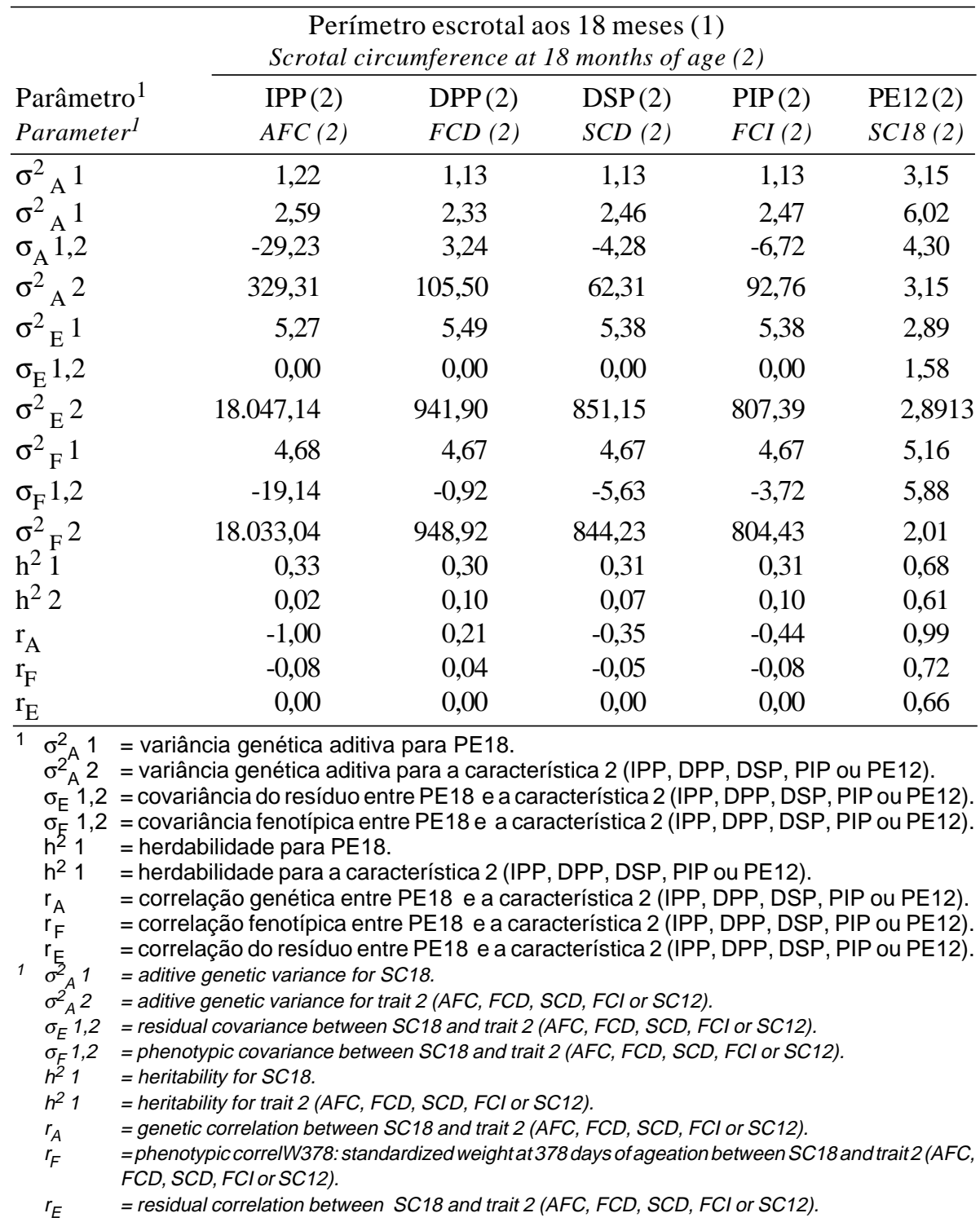

tros escrotais estariam associados à diminuição na data do segundo parto, levando à redução no primeiro intervalo de partos. A correlação foi negativa e de pequena magnitude entre o perímetro escrotal medido aos 12 meses e a data do primeiro parto, estando o maior perímetro escrotal associado à menor data do primeiro parto e ao aumento do primeiro intervalode partos. Já a correlação entre a data do segundo parto e o perímetro escrotal aos 12 meses foi elevada e negativa, indicando que maiores perímetros escrotais estariam associados à tendência de diminuição do primeiro intervalo de partos.

Alguns resultados aparentemente contraditórios foram encontrados entre as características perímetro escrotal e data do parto (Tabelas 2 e 3). Embora as correlações genéticas entre o perímetro escrotal aos 12 meses de idade e as duas datas do parto 
tenham sido favoráveis, maior associação genética foi verificada para a segunda data do parto $(-0,71)$ que para a primeira $(-0,08)$. Como enfatizado anteriormente, o interesse da inclusão da característica perímetro escrotal nos programas de seleção estaria relacionado, entre outros fatores, a sua favorável associação genética com a idade à puberdade e com o início da atividade reprodutiva dos animais (BOURDON e BRINKS 1986; NOTTER, 1988). Como nas fêmeas bovinas, essas características estão mais relacionadas com a data do primeiro parto, seria lógico esperar maior estimativa para a correlação envolvendo a primeira data do parto. Esta aparente inversão encontra suporte na literatura. Estimando as correlações genéticas entre perímetro escrotal de touros ao ano de idade e às datas do primeiro e segundo partos de fêmeas da raça Angus, NOTTER et al. (1993) obtiveram resultado semelhantes ao deste trabalho $(-0,19$ e $-0,27$, respectivamente, para as correlações entre o perímetro escrotal ao 12 meses de idade e a data do primeiro e segundo partos). Os autores argumentaram que o perímetro escrotal de touros jovens estaria associado a aspectos reprodutivos das fêmeas bovinas que, aparentemente, ultrapassam a idade à puberdade.

As estimativas de correlação genética entre datas dos partos e o perímetro escrotal aos 18 meses de idade apresentaram-se ainda mais inesperadas. O valor obtido para a correlação envolvendo a data do segundo parto foi favorável $(-0,35)$, enquanto para a data do primeiro parto o sentido foi desfavorável $(0,21)$. Este fato encontra algum suporte na literatura, quando MORRIS e CULLEN (1994) encontraram correlações genéticas positivas entre o perímetro escrotal e a data do parto.

A propósito da aparente inconsistência deste último resultado, algumas hipóteses podem ser aventadas à luz das intrínsecas associações entre precocidade sexual, tamanho e peso corporal e perímetro escrotal. Diversos estudos realizados na década de 70 enfatizam diferenças entre raças e indivíduos dentro de raças, quanto ao tamanho corporal, à precocidade sexual e eficiência produtiva dos animais em diversos sistemas de criação. Por exemplo, DICKERSON (1978) sustenta que, quando ocorrem limitações de ordem nutricional, como as prevalentes nos sistemas extensivos de criação das regiões tropicais, os indivíduos geneticamente de menor tamanho dentro de determinada raça seriam mais precoces, entrando em reprodução e atingindo peso à maturidade mais cedo. Estes animais seriam menos exigentes em requerimentos nutricionais para mantença que animais maiores, sendo mais férteis e adaptados às condições de estresse alimentar (FITZHUGH, 1978, entre outros). No Brasil, MARIANTE eZANCANER (1985) e BARBOSA (1991) encontraram evidências de antagonismo genético entre crescimento e reprodução de fêmeas em ambiente desfavorável. Além disso, MERCADANTE (1995) reportou correlações genéticas próximas de zero, mas desfavoráveis, entre peso aos 240 e 365 dias e o primeiro intervalo de partos. Mais recentemente, JOHNSTON e BUNTER (1996), analisando informações de animais Angus, concluíram que dias para o primeiro parto (semelhante à data do primeiro parto) apresentou correlação genética desfavorável com os pesos à desmama e ao ano de idade. Esses autores comentaram que os animais com maior potencial genético para crescimento corporal teriam desvantagens reprodutivas, quando do primeiro acasalamento para as condições australianas.

O fato de que a precocidade sexual esteja associada a taxas de crescimento corporal relativo em idades jovens, evidenciando a importância das diferenças entre curvas de crescimento dos animais, ficou demonstrado no trabalho de BERGMANN e HOHENBOKEN (1992), no qual novilhas Angus que possuíam menores taxas de crescimento relativo entre a desmama e um ano de idade (animais mais precoces) foram as que apresentaram maior fertilidade quando entraram em reprodução aos 13-14 meses de idade.

Para a raça Nelore, o crescimento do perímetro escrotal apresentou-se de forma linear até aos 12 meses de idade, com tendência à curvilinearidade após essa idade e redução do crescimento próxima aos 18 meses de idade (BERGMANN et al., 1996, 1998). QUIRINO et al. (1999) descreveram a curva de crescimento do perímetro escrotal, com ponto de inflexão (máximo crescimento) aos 13,1 meses de idade. Segundo esses autores, esse fato evidenciaria o maior crescimento do parênquima testicular, ocorrendo próximo ao ano de idade, sugerindo o início do período pré-pubere. Segundo FITZHUGH (1976), nesse ponto da curva de crescimento, a taxa de crescimento é máxima, passando de função crescente (autoacelerante) para função decrescente (autoinibitória). Para FOOT (1969), o início da puberdade é caracterizado pelo crescimento rápido das gônadas e pode ser determinado pela presença de espermatozóides viáveis no ejaculado, associado à liberação do pênis e da libido, com o estabelecimento 
da capacidade reprodutiva.

As poucas pesquisas realizadas no Brasil considerando aspectos relacionados à puberdade de animais zebus salientam sua maior dependência do estado corporal do que da idade. Diversos trabalhos sugerem idades variando entre 10 e 12 meses de idade para o aparecimento da puberdade de machos zebus, coincidindo com o período de grande ganho de peso e associado ao rápido crescimento testicular, aumento da secreção de LH e início da espermatogênese (CARDOSO, 1977; CASTRO et al., 1989). Paralelamente, o perímetro escrotal tem sido preconizado como característica indicadora da puberdade em machos e nas fêmeas que apresentam algum parentesco com eles. Existe suporte na literatura para se concluir que os fatores hormonais que promovem o desenvolvimento testicular inicial nos machos são os mesmos que promovem o desenvolvimento ovariano inicial nas fêmeas, e a seleção para a puberdade precoce em um sexo resultará em associada redução da idade a puberdade do outro sexo (MACKINNON et al., 1990).

Embora o perímetro escrotal esteja associado ao peso corporal (BERGMANN et al., 1996, entre outros), segundo TOELLE e ROBSON (1985), existem evidências de que o tamanho do testículo seja controlado por diferentes sistemas fisiológicos nas idades de 205 e 365 dias de taurinos criados em condições temperadas. Segundo esses autores, o perímetro escrotal medido aos 205 dias seria reflexo do aumento do peso corporal e, entre 205 e 365 dias, os testículos passariam a ser influenciados pelos hormônios gonadotrópicos. Dessa forma, as diferenças entre perímetros escrotais aos 365 dias de idade estariam mais dependentes das diferenças relacionadas ao sistema hormonal do que ao peso corporal.

Conforme salientado anteriormente, existem evidências na literatura de que a puberdade de machos Nelore em condições nutricionais adequadas, comoé o caso do presente estudo, ocorre próxima ao ano de idade. Portanto, os resultados do presente estudos, relativos às correlações genéticas entre perímetro escrotal e data do primeiro parto, indicam que a seleção de animais com maior perímetro escrotal aos 12 meses de idade estaria associada à escolha de animais que apresentassem maiores níveis de hormônios gonadotrópicos, culminando com a puberdade, o início de atividade reprodutiva das fêmeas, as concepções mais precoces na primeira estação de monta e menores datas do primeiro parto. Aos 18 meses de idade, a maioria dos animais já estaria em período pós-pubere e a seleção praticada para maiores perímetros escrotais nessa idade estaria associada a maiores pesos corporais e, possivelmente, à menor precocidade reprodutiva em ambientes tropicais.

Esta vantagem seletiva da característica perímetro escrotal aos 12 meses sobre 18 meses de idade contradiz as indicações de BERGMANN et al. (1996) e QUIRINO e BERGMANN (1998), os quais recomendam seleção para perímetro escrotal realizada aos 18 meses de idade. Entretanto, esses autores não consideraram os reflexos desta seleção sobre características reprodutivas das fêmeas.

Finalmente, deve-se salientar a possibilidade de que estas estimativas de correlações genéticas estejam associadas a substanciais erros-padrão (KOOT et al., 1994b). Também, de acordo com MEYER (1991), para análises realizadas com modelo animal envolvendo características diferentes e mensuradas em diferentes animais, como é o caso do perímetro escrotal e das características reprodutivas das fêmeas, deve-se ter em mente que as informações referentes às covariancias genéticas são resgatadas apenas por meio da relação de parentesco entre os animais. Portanto, estas estimativas estariam sujeitas a grandes erros-padrão, a menos que os animais apresentem estreita relação de parentesco, ou ainda que o volume de informações seja substancial.

\section{Conclusões}

As correlações genéticas estimadas entre perímetro escrotal e características observadas nas fêmeas foram favoráveis, variando apenas em magnitude. Entretanto, a correlação genética entre o perímetro escrotal medido aos 18 meses de idade e a data do primeiro parto foi desfavorável. Por outro lado, o perímetro escrotal medido aos 12 meses apresentou correlação genética favorável com a data do primeiro parto. Dessa forma, nas condições desse estudo, a seleção do perímetro escrotal medido aos 12 meses seria mais efetiva que a seleção do perímetro medido aos 18 meses de idade, quando se deseja obter melhoria relacionada às características reprodutivas das fêmeas. Os resultados desse trabalho, para os parâmetros genéticos, indicam que é possível obter incremento das características reprodutivas das fêmeas via seleção para aumento do perímetro escrotal nos machos, e a idade mais apropriada para a seleção para perímetro escrotal seria aos 12 meses de idade, ao contrário das recomendações descritas na literatura nacional, que enfatizam a seleção do perímetro 
escrotal aos 18 meses de idade.

Deve-se ressaltar que, devido às peculiaridades desse material, de localidade e das circunstâncias do período estudado, as aplicações de ordem prática dos resultados encontrados não devem ser generalizadas a outros rebanhos, sem análise mais detalhada dos fatores envolvidos. São necessários mais estudos relacionados com os parâmetros genéticos envolvendo o perímetro escrotal e as características reprodutivas das fêmeas, sugerindo-se a adoção de penalizações para as fêmeas que falharam em conceber.

\section{Agradecimento}

À Colonial Agropecuária Ltda., pela cessão das informações.

Ao Conselho Nacional de Desenvolvimento Científico e Tecnológico (CNPq), à Fundação de Amparo a Pesquisa do Estado de Minas Gerais (FAPEMIG), à Pró-Reitoria de Pesquisa da UFMG (PRPq-UFMG) e à Fundação de Estudo e Pesquisa em Medicina Veterinária e Zootecnia da Escola de Veterinária da UFMG (FEP/MVZ), pelo suporte financeiro para viabilizar o estudo.

\section{Referências Bibliográficas}

ANDRADE, V.J. 1991. Manejo alternativo da reprodução em bovinos de corte. Cad. Téc. Esc. Vet, 6:29-54.

BARBOSA, P.F. Análise genético-quantitativa de características de crescimento e reprodução em fêmeas da raça Canchim. Ribeirão Preto:USP, 1991.237p. Tese (Doutorado em Genética) - Faculdade de Medicina de Ribeirão Preto/Universidade de São Paulo, 1991.

BERGMANN, J.A.G., GRESSLER, S. L., PEREIRA, C. S. et al. 1998. Avaliação de fatores genéticos e de ambiente sobre deferentes características reprodutivas de fêmeas da raça Nelore em regime de estação de monta restrita. Arq. Bras. Med. Vet. Zootec., 50(5):633-645.

BERGMANN, J.A.G. 1993. Melhoramento genético da eficiência reprodutiva em bovinos de corte. Rev. Bras. Reprod. Anim. 1(4):70-86. (Suppl).

BERGMANN, J.A.G., HOHENBOKEN, W.D. 1992. Prediction of fertility from calfhood traits of Angus and Simmental heifers. J. Anim. Sci., 70(9):2611-2621.

BERGMANN, J.A.G., ZAMBORLINI, L.C., PROCÓPIO, C.S.O. et al. 1996. Estimativas de parâmetros genéticos do perímetro escrotal e do peso corporal em animais da raça Nelore. Arq. Bras. Med. Vet. Zootec., 48(1):69-78.

BOLDMAN, K.G., KRIESE, L.A., VAN VLECK, L.D. et al. 1995. A manual for use for MTDFREML. A set of programs to obtain estimates of variance and covariance [DRAFT]. Lincoln, Department of Agricultural/Agricultural Research Service. $120 \mathrm{p}$.

BOURDON, R.M., BRINKS, J.S. 1983. Calving date versus calving interval as a reproductive measure in beef cattle. $J$. Anim. Sci., 57(6):1412-1417.
BOURDON, R.M., BRINKS, J.S. 1986. Scrotal circumference in yearling Hereford bulls: adjustment factors, herdabilities and genetic, environmental and phenotypic relationships with growth traits. J. Anim. Sci., 62(4):958-967.

CARDOSO, F.M. Desenvolvimento dos orgãos genitais masculinos de Zebus (Bos indicus), da raça Nelore, do período fetal aos 36 meses de idade. Belo Horizonte, UFMG. 1997. 113p. Dissertação (Mestrado em Medicina Veterinária) - Escola de Veterinária/Universidade Federal de Minas Gerais, 1977.

CASTRO, V.M., VALE FILHO, V.R., REIS, S.R. et al. 1989. Puberdade e início de maturação sexual em touros Nelore, de 10-14 meses de idade. Rev. Bras. Reprod. Anim, Supl., 1:183.

DICKERSON, G.E. 1978. Animal size and efficiency: basic concepts. Anim. Prod., 27(3):367-379.

FALCONER, D.S. 1989. Introduction to quantitative genetics. 3.ed. New York: John Wiley \& Sons, Inc., New York. 438p.

FITZHUGH, H.A. 1976. Analysis of growth curves and strategies for altering their shape. J. Anim. Sci., 30(5):706-711.

FITZHUGH, H.A. 1978. Animal size and eficiency, with special reference to the breeding female. Anim. Prod., 27(3):393-401.

FOOT, R.H. 1969. Physiological aspect of artificial insemination. In: COLE, H.H., CUPPS, P. (Eds.) Reproduction in domestic animals. New York: Academic Press 2.ed. p.313-353.

GRESSLER, S.L. Estudo de fatores de ambiente e parâmetros genéticos de algumas características reprodutivas em animais da raça Nelore. Belo Horizonte: UFMG, 1998. 149p. Dissertação (Mestrado em Zootecnia) - Escola de Veterinária/Universidade Federal de Minas Gerais, 1998.

JOHNSTON, D.J., BUNTER, K.L. 1996. Days to calving in Angus cattle: genetic and environmental effects, and covariances with other traits. Lvstck. Prod. Sci., 45(1):13-22.

KOOTS, K.R., GIBSON, J.P., SMITH, C. et al. 1994a. Analyses of published genetic parameters estimates for beef production traits. 1-Herdability. Anim. Breed. Abstr., 62(5):309-338.

KOOTS, K.R., GIBSON, J.P., WILTON, J.W. 1994b. Analyses of published genetic parameters estimates for beef production traits. 2-Phenotypic and genetics correlations. Anim. Breed. Abstr., 62(11):825-853.

LÔBO, D.B. 1996. Programa de melhoramento genético da raça Nelore. Universidade de São Paulo, Ribeirão Preto. p.77.

LÔBO, R.B., REYES, A., FERRAZ, J.B.S. Bivariate animal analysis of growth weights and scrotal ciecunference of Nellore cattle in Brazil. In: WORLD CONGRESS ON GENETICS APPLIED TO LIVESTOCK PRODUCTION, 5, 1994, Guelph, Canada. Proceedings...Guelph, Canada, 1994, p.199-202.

MACKINNON, M.J., TAYLOR, J.F., HETZEL, D.J.S. 1990. Genetic variation and covariation in beef cow and bull fertifity. J. Anim. Sci. 68(5):1208-1214.

MARIANTE, A.S., ZANCANER, A. 1985. Crescimento $e$ reprodução em gado Nelore. Visão do criador e do pesquisador. 1 ed., São Paulo: Ed. dos Criadores. 152p.

MARTINS-FILHO, R. Estimativas de correlação genéticas entre circunferência escrotal em bovinos da raça Nelore e características reprodutivas em suas meias-irmãs paternas. Ribeirão Preto, SP: USP, 1991. 92p. Tese (Doutorado em Genética) - Faculdade de Medicina de Ribeirão Preto, Universidade de São Paulo. 1991.

MEACHAM, N.S., NOTTER, D.R. 1987. Heritability estimates for calving date in simental cattle. J. Anim. Sci., 64(3):701-705.

MERCADANTE, M.E.Z. Estudo das relações genético-quantitativas entre características de reprodução, crescimento e produção em fêmeas da raça Nelore. Ribeirão Preto: USP, 1995.90p. Dissertação (Mestrado em Genética)-Faculdade de Medicina de Ribeirão Preto, Universidade de São Paulo. 1995. 
MEYER, K. 1991. Estimating variances and covariances for multivariate animal models by restricted maximum likelihood. Genet Sel. Evol., 23(1):67-83.

MEYER, K., HAMMOND, K., MACKINNON, M.J. et al. 1991. Estimates of covariances between reproduction and growth in australian beef cattle. J. Anim. Sci., 69(9):3533-3543.

MEYER, K., HAMMOND, K., PAMELL, P.F., et al. 1990. Estimates of heritability and repeatability for reproductive traits in Australian beef cattle. Lvstck. Prod. Sci., 25(1):15-30.

MILAGRES, J.C., DILLARD, E.U., ROBISON, O.W. 1979. Heritability estimates for some measures of reproduction in Hereford heifers. J. Anim. Sci., 49(3):668-674.

MORRIS, C.A., CULLEN, N.G. 1988. Oestrous and reproductive performance of early and late calving beef cows. N.Z. J. Agric. Res., 31(4):395-399.

MORRIS, C.A., CULLEN, N.G.A. 1994. Note on genetic correlation between pubertal traits of males or females and liftime pregnancy rate in beef cattle. Lvstck. Prod. Sci., 39(3):291-297.

MORRIS, C. A. Theoretical and realised responses to selection for reproductive rate. In: WORLD CONGRESS ON GENETICS APPLIED TO LIVESTOCK PRODUCTION, 4, 1990, Edinburgh, Scotland. Proceedings... Edinburgh, Scotland, 1990, p.309-318.

NOTTER, D.R. Evaluating and reporting reprodutive traits. In: BEEF IMPROVEMENT FEDERATION, 20, 1988, Albuquerque, New Mexico, Proceedings... Albuquerque, New Mexico, 1988. p.21-42.

NOTTER, D.R. 1995. Maximizing fertility in animal breeding programs. Blacksburg, Virginia Polytechnic Institute e State University, Department of Animal and Poultry Sciences. p. inreg. (XI Congresso Brasileiro de Reprodução Animal . Belo Horizonte - pré-congresso).

NOTTER, D.R., MC FADDEN, L.G., BERGMANN, J.A.G. Relationship between yearling scrotal circumference and measures of femeale reproduction in Angus cattle. In: BEEF IMPROVEMENT FEDERATION, 25, 1993, Asheville, North Carolina, Proceedings... Asheville, North Carolina, 1993. p.180-184.
NOTTER, D.R., JOHNSON, M.H. 1987. Simulation of genetic control of reproduction in beef cows. III. within-herd breeding values estimation with known breeding dates. J. Anim. Sci., 65(1):88-98.

NOTTER, D.R., JOHNSON, M.H. 1988. Simulation of genetic control of reproduction in beef cows. IV. within-herd breeding values estimation with pasture mating. J. Anim. Sci., 66(2):280-286.

PONZONI, R.W., GIFFORD, D.R. 1994. Reproductive and some peri-natal variables in a mixed breed beef cattle herd. J. Anim. Breed. Gen., 111(1):52-64.

PONZONI, R.W. 1992. Which trait for genetic improvement of beef cattle reproduction: calving rate or calving day. J. Anim. Breed. Gen., 10(2):119-128.

QUIRINO, C.R., BERGMANN, J.A.G. 1998. Heritability of scrotal circumference adjusted and unadjusted for body weight in Nellore bulls using ani and bivariate animal models. Theroigenology, 48(7):1398-1396.

QUIRINO, C.R., BERGMANN, J.A.G., VALE FILHO, V.R. et al. 1999. Evaluation of four mathematical functions to describe scrotal circumference maturation in Nellore bulls. Theroigenology, (no prelo).

SAS Institute, SAS (Statistical Analysis System). User's Guide. SAS Institute Inc., Cary, NC, 1990.

TOELLE, V.D., ROBISON, O.W. 1985. Estimates of genetic correlations between testicular measurements and female reproductive traits in cattle. J. Anim. Sci., 60(1):89-100.

Recebido em: 12/05/99

Aceito em: 26/08/99 